\title{
Age- and sex-related characteristics of the increasing trend of nontuberculous mycobacteria pulmonary disease in a tertiary hospital in South Korea from 2006 to 2016
}

\author{
Youngmok Park ${ }^{1,}$, Chi Young Kim ${ }^{2,}$, Moo Suk Park ${ }^{1}$, Young Sam Kim ${ }^{1}$, Joon Chang ${ }^{1}$, and Young Ae Kang ${ }^{1}$
}

${ }^{1}$ Division of Pulmonology, Department of Internal Medicine, Severance Hospital, Yonsei University College of Medicine, Seoul; ${ }^{2}$ Division of Pulmonology, Department of Internal Medicine, Korea University Ansan Hospital, Ansan, Korea

\author{
Received: November 22, 2019 \\ Revised : February 17, 2020 \\ Accepted: February 27, 2020

\section{Correspondence to} \\ Young Ae Kang, M.D. \\ Division of Pulmonology, \\ Department of Internal \\ Medicine, Severance Hospital, \\ Yonsei University College \\ of Medicine, 50-1 Yonsei-ro, \\ Seodaemun-gu, Seoul 03722, \\ Korea \\ Tel: +82-2-2228-1954 \\ Fax: +82-2-393-6884 \\ E-mail: mdkang@yuhs.ac \\ https://orcid.org/0000-0002- \\ 7783-5271 \\ ${ }^{*}$ These authors contributed
equally to this work.
}

Background/Aims: The incidence rate of nontuberculous mycobacterial pulmonary disease (NTM-PD) is increasing worldwide. However, data regarding the age- and sex-specific epidemiology of NTM-PD are limited. This study aimed to investigate the long-term epidemiologic trends of NTM-PD within the recent 11year period in a tertiary referral hospital in Korea.

Methods: We retrospectively reviewed the medical records of the patients diagnosed with NTM-PD between January 2006 and December 2016 at Severance Hospital, South Korea.

Results: There were 1,017 incident cases with NTM-PD during the study period. The mean age was 62.7 years, and $41.2 \%$ were men. Women were younger than men (59.9 years vs. 66.7 years, $p<0.001$ ) and a higher proportion of women had bronchiectasis ( $88.6 \%$ vs. $77.1 \%, p<0.001$ ). The incidence rates of NTM-PD annually increased by $14 \%$ (95\% confidence interval, 10\% to 19\%) from 1.2 in 2006 to 4.8 in 2016 (per 100,000 patients-year). The peak incidence rate was in the 50 s for women and in the 70 for men, except for those aged $\geq 80$ years. Mycobacterium avium complex was the most common causative species of NTM-PD (63.6\%).

Conclusions: The incidence rate of NTM-PD in a tertiary referral hospital in South Korea continued to increase from 2006 to 2016. Furthermore, there were age- and sex-related differences in the clinical characteristics, which might contribute to understanding the nature of the disease and inherited and acquired host factors.

Keywords: Nontuberculous mycobacteria; Nontuberculous mycobacterium infection; Lung diseases

\section{INTRODUCTION}

Nontuberculous mycobacteria (NTM) represent a diverse group of mycobacterial species; this complex differs from the Mycobacterium tuberculosis complex and Mycobacterium leprae. NTM are ubiquitous environmen- tal bacteria and are mainly present in the natural and drinking water systems, pools and hot tubs, biofilms, and soil [1]. They can affect various tissues and body fluids, causing pulmonary and extrapulmonary diseases $[2,3]$. Although NTM are considered opportunistic pathogens, the incidence and prevalence rate of NTM 
pulmonary disease (NTM-PD) are increasing worldwide, not only in the immune-compromised, but also in the immune-competent patients [2-4]. Furthermore, there are significant geographic differences in the distribution of NTM primarily due to the environmental nature of these microorganisms [5].

In South Korea, a country with an intermediate tuberculosis (TB) burden [6], NTM are also emergent pathogens, and NTM-PD is rapidly becoming a significant public health concern. Several studies have reported an increase in both the NTM recovery rate in clinical specimens and the incidence and prevalence rate of NTM-PD in South Korea [7-13]. However, comprehensive analyses of the clinical and epidemiologic data for NTM-PD are relatively scarce compared to that of TB because NTMPD is not a reportable disease in most countries, and complicated criteria and extensive follow-up are required for diagnosis.

We had previously reported the change in the NTM epidemiology in a tertiary referral hospital in South Korea between 2006 and 2010 [7]. In this study, we aimed to investigate the long-term epidemiologic trends of NTM-PD within the recent 11-year period in a tertiary referral hospital in South Korea. Moreover, we intend to perform comprehensive analyses of NTM-PD and its association with age and sex.

\section{METHODS}

\section{Study design and data collection}

We retrospectively analyzed the mycobacterial culture records at Severance Hospital, a tertiary referral hospital in Seoul, South Korea, from January 2006 to December 2016. The culture results from the pulmonary specimens were included, and those from the extrapulmonary samples were excluded. NTM-PD was defined according to the American Thoracic Society/Infectious Disease Society of America (ATS/IDSA) criteria [2]. An incident case of NTM-PD in a specific year was defined as the year of the first recovery date of NTM in a respiratory specimen. The data on age, sex, body mass index, comorbidities, and spirometry results within 6 months from the first recovery date of each patient were retrospectively obtained from the medical records.

\section{Microbiological examination}

Respiratory specimens, such as sputum and bronchial washing fluid, were stained according to the ATS/IDSA guidelines [2], decontaminated with $4 \% \mathrm{NaOH}$, homogenized, and concentrated via centrifugation. Liquid medium (mycobacteria growth-indicator tube) has been used in Severance Hospital since 2008. The detailed information regarding specimen processing has been published previously [7]. We identified the NTM species with polymerase chain reaction-restriction fragment length polymorphism analysis based on the rpoB gene [14].

\section{Causative species of NTM-PD}

We defined the causative species of NTM-PD as the NTM species identified two or more times in the sputum or once in the bronchial wash or lavage. If there are two or more causative species of NTM-PD, we defined the disease as multi-species NTM-PD.

\section{Radiologic evaluation}

The radiologic patterns of NTM-PD were classified into three groups according to the chest radiography and computed tomography (CT) findings [2]. The fibrocavitary (FC) form was defined as the presence of cavitary opacities in the upper lobes, either with or without consolidation and pleural thickening. The nodular bronchiectatic (NB) form was defined as bilateral bronchiectasis with multiple nodules and tree-in-bud opacities. The unspecified form was defined as the absence of evident characteristics of either form.

\section{Incidence rate of NTM-PD}

The incidence rate of NTM-PD was calculated as the number of patients with NTM-PD divided by the annual total number of patients who visited Severance Hospital. We also investigated the age group- and sex-specific incidence rates, detection rates of each causative species, and the female-to-male rate ratio of NTM-PD.

\section{Statistical analysis}

The categorical variables were analyzed with Pearson's chi-squared test or Fisher's exact test, while the continuous variables were analyzed with Student's $t$ test. A Cochran-Armitage trend test was used for the analysis of the changing trends, and Poisson regression models with allowance for overdispersion were fit to the collect- 
ed data for the analysis of the incidence rate change. The fitted model was used to calculate the annual percent change in the incidence rate. All statistical analyses were performed using R v3.6.o (R Foundation for Statistical Computing, Vienna, Austria), and a two-tailed $p<0.05$ was considered statistically significant.

\section{Ethics statement}

The study protocol was reviewed and approved by the Institutional Review Board of Severance Hospital (No. 4-2018-0350), and the need for obtaining informed consent was waived by the committee due to the retrospective nature.

\section{RESULTS}

\section{Respiratory specimens and patients}

We reviewed 306,008 respiratory samples from 64,627 patients during the study period. NTM isolates were recovered in 17,485 (5.7\%) specimens, and the recovery rate (95\% confidence interval [CI]) increased from 1.9\% (95\% CI, $1.7 \%$ to $2.2 \%$ ) in 2006 to $7.4 \%$ (95\% CI, $7.2 \%$ to $7.7 \%$ ) in 2016 ( $p$ for trend < 0.001 ). NTM were newly observed in 3,843 patients during the study period, of whom 1,017 (26.5\%) patients satisfied the criteria for NTM-PD. The annual number of incident cases of NTM-PD and patients who visited this institution are presented in Supplementary Table 1.

\section{Clinical characteristics and incidence rate of NTM- PD according to the sex and age}

The baseline characteristics of the patients with NTM$\mathrm{PD}$ are shown in Table 1. Women were younger than men. The rates of bronchiectasis and NB form were higher among women than that among men. The proportion of patients with a history of pulmonary TB or chronic obstructive pulmonary disease (COPD) was higher among men than among women.

Fig. 1 shows the age distribution of the patients with NTM-PD. Most male patients were in their 6os and 70s, whereas most female patients were in their 50 and early 6os. The incidence rate of patients with NTMPD showed a similar distribution according to the age group (Supplementary Table 2). The peak incidence rate of NTM-PD was in the 5os for women and in the 70 s for men, except for those aged $\geq 80$ years. The overall incidence rate increased as age increased for both sexes, with male patients showing an 83\% (95\% CI, $57 \%$ to $117 \%$ ) increase in the overall incidence rate, and female patients showing a $47 \%$ (95\% CI, $20 \%$ to $86 \%$ ) increase. The female-to-male incidence rate ratio was lower than 1 for patients aged $\geq 70$ years (Supplementary Table 2).

Fig. 2A shows the sex-specific incidence rate for patients with NTM isolated from the respiratory specimens and patients with NTM-PD in each year from 2006 to 2016. The incidence rate of the isolated NTM was 4.6 (95\% CI, 3.7 to 5.6) in 2006 and increased to 19.6 (95\% CI, 18.0 to 21.3 ) in 2016 ( $p$ for trend < 0.001 ), while the incidence rate of NTM-PD was 1.2 (95\% CI, o.8 to 1.8) in 2006 and increased to 4.8 (95\% CI, 4.1 to 5.7 ) in 2016 ( $p$ for trend $<0.001$ ). Poisson regression analysis showed that the incidence rate of NTM-PD increased annually by $14 \%$ (95\% CI, $10 \%$ to $19 \%$ ) in the total population. Fig. 2B shows the age group-specific incidence rate of NTM-PD. The increasing trend of NTM-PD was more prominent in the older population (annual percentage change-in the $40 \mathrm{~S}, 6 \%$ [ $95 \% \mathrm{CI},-1 \%$ to $14 \%$ ]; in the $50 \mathrm{~S}$, $9 \%$ [ $95 \% \mathrm{CI}, 4 \%$ to $15 \%$ ]; in the $60 \mathrm{os}, 12 \%$ [95\% CI, $7 \%$ to $18 \%$ ]; in the $70 \mathrm{Os}, 17 \%$ [ $95 \% \mathrm{CI}, 10 \%$ to $25 \%$ ]; and in the 8os: $17 \%$ [95\% CI, $6 \%$ to $30 \%]$ ).

\section{Causative species of NTM-PD}

The most commonly isolated NTM species from the patients with NTM-PD were M. avium complex (MAC, M. avium and M. intracellulare), followed by M. abscessus complex (MABC, M. abscessus and M. massiliense) (Table 2). Multi-species NTM-PD was identified in $20.2 \%$ of the patients, and the rate of multi-species NTM-PD was higher among women than among men. M. kansasii infection was more common among the male patients and in those with the FC form disease.

Fig. 3 shows the causative species of NTM-PD during the study period. Notably, the most predominant causative species was MAC, and the proportion showed no significant variation for 11 years $(p$ for trend $=0.072$ ). MABC tended to decrease over time ( $p$ for trend $=0.035$ ). These findings suggest that the increase in the incidence rate of NTM-PD is mainly because of the increase in MAC.

\section{Radiologic forms of NTM-PD}

The predominant radiologic findings of NTM-PD were 
Table 1. Characteristics of the patients with nontuberculous mycobacterial pulmonary disease

\begin{tabular}{|c|c|c|c|c|}
\hline Characteristic & $\operatorname{Total}(\mathrm{n}=1,017)$ & Male $(\mathrm{n}=419)$ & Female $(n=598)$ & p value \\
\hline Age, yr & $62.7 \pm 11.7$ & $66.7 \pm 10.8$ & $59.9 \pm 11.6$ & $<0.001$ \\
\hline Body mass index, $\mathrm{kg} / \mathrm{m}^{2}$ & $20.5 \pm 4.1$ & $20.6 \pm 4.8$ & $20.4 \pm 3.4$ & 0.448 \\
\hline Smoking status & & & & $<0.001$ \\
\hline Never smoker & $731(73.0)$ & $164(39 \cdot 3)$ & $567(96.9)$ & \\
\hline Ever smoker & $271(27.0)$ & $253(60.7)$ & $18(3.1)$ & \\
\hline Pack-year & $28.6 \pm 21.6$ & $29.1 \pm 22.1$ & $20.4 \pm 11.4$ & 0.024 \\
\hline Previous tuberculosis & $491(48.3)$ & $238(56.8)$ & $253(42.3)$ & $<0.001$ \\
\hline \multicolumn{5}{|l|}{ Comorbidity } \\
\hline Bronchiectasis & $853(83.9)$ & $323(77.1)$ & $530(88.6)$ & $<0.001$ \\
\hline COPD & $137(13.5)$ & $102(24 \cdot 3)$ & $35(5.9)$ & $<0.001$ \\
\hline Asthma & $74(7.3)$ & $28(6.7)$ & $46(7.7)$ & 0.626 \\
\hline Lung cancer & $41(4.0)$ & $19(4.5)$ & $22(3.7)$ & 0.602 \\
\hline Other pulmonary diseases ${ }^{\mathrm{a}}$ & $21(2.1)$ & $7(1.7)$ & $14(2.3)$ & 0.606 \\
\hline Malignancy, other than lung & $238(23.4)$ & $116(27.7)$ & $122(20.4)$ & 0.009 \\
\hline Diabetes mellitus & $137(13.5)$ & $75(17.9)$ & $62(10.4)$ & 0.001 \\
\hline Transplantation & $16(1.6)$ & $12(2.9)$ & $4(0.7)$ & 0.012 \\
\hline Immune-compromised & $5(0.5)$ & $2(0.5)$ & $3(0.5)$ & $>0.999$ \\
\hline No comorbidity ${ }^{\mathrm{b}}$ & $13(1.3)$ & $6(1.4)$ & $7(1.2)$ & 0.935 \\
\hline Radiologic pattern & & & & $<0.001$ \\
\hline Fibrocavitary form & $103(10.1)$ & $65(15.5)$ & $38(6.4)$ & \\
\hline Nodular bronchiectatic form & $831(81.7)$ & $309(73.7)$ & $522(87.3)$ & \\
\hline Unspecified & $83(8.2)$ & $45(10.7)$ & $38(6.4)$ & \\
\hline Lung function & 344 & 173 & 171 & \\
\hline FVC, \% predicted & $80.9 \pm 21.8$ & $74.6 \pm 21.6$ & $87.3 \pm 20.2$ & $<0.001$ \\
\hline $\mathrm{FEV}_{1}, \%$ predicted & $85.6 \pm 49.9$ & $81.6 \pm 66.1$ & $89.7 \pm 23.8$ & 0.129 \\
\hline $\mathrm{FEV}_{1} / \mathrm{FVC}$ & $72.5 \pm 13.2$ & $70.5 \pm 15.4$ & $74.5 \pm 10.2$ & 0.005 \\
\hline
\end{tabular}

Values are presented as mean $\pm \mathrm{SD}$ or number (\%).

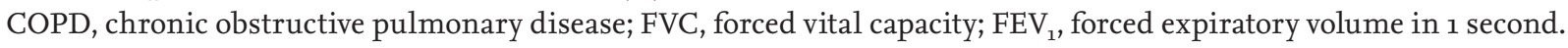

${ }^{a}$ Pulmonary disease other than tuberculosis, COPD, asthma, bronchiectasis, and lung cancer.

${ }^{\mathrm{b}}$ Patients without any comorbidities listed in this table.

NB form, accounting for an average of $78 \%$ of the cases; this further increased over time ( $p$ for trend $<0.001$ ) (Fig. 4). This was followed by the FC form (14\%), but the proportion of patients with the FC form decreased ( $p$ for trend $<0.001$ ). The average proportion of patients with the NB form was higher among women than among men ( $85 \%$ vs. $69 \%$ ), but similar patterns of increased proportion of the NB form and decreased proportion of the FC form were found among the male and female subgroups ( $p$ for trend $<0.001$ for both) (Supplementary Fig. 1).

\section{DISCUSSION}

This study found that the incidence rate of NTM-PD continued to increase between 2006 and 2016 in a tertiary hospital in South Korea. Moreover, there were some differences in the clinical characteristics of the patients with NTM-PD according to age, sex, and radiologic forms of the disease.

An increased rate of isolated NTM and NTM-PD has been reported worldwide. In Korea, the incidence rate of NTM continues to increase despite no significant 


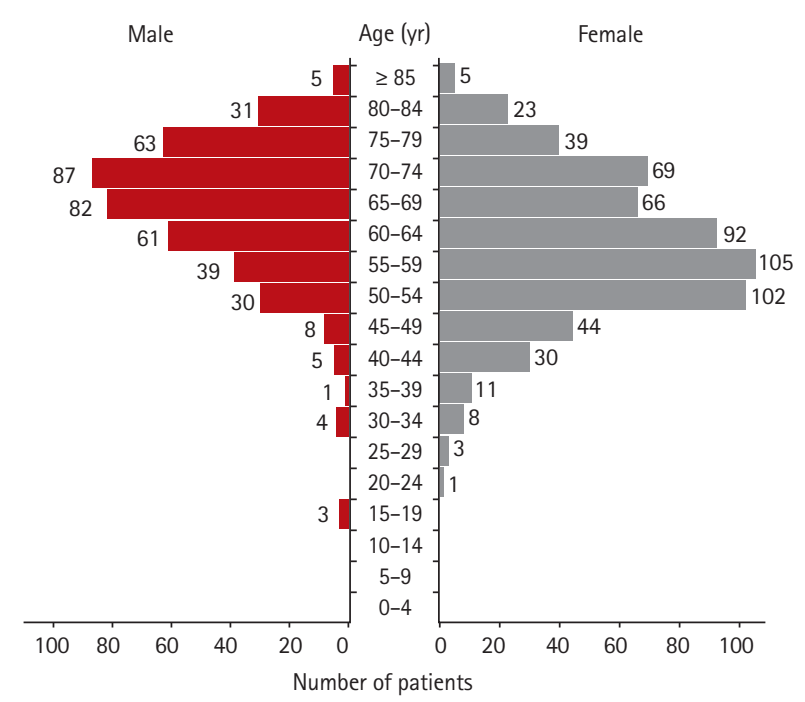

Figure 1. Age distribution of the patients with nontuberculous mycobacterial pulmonary disease.

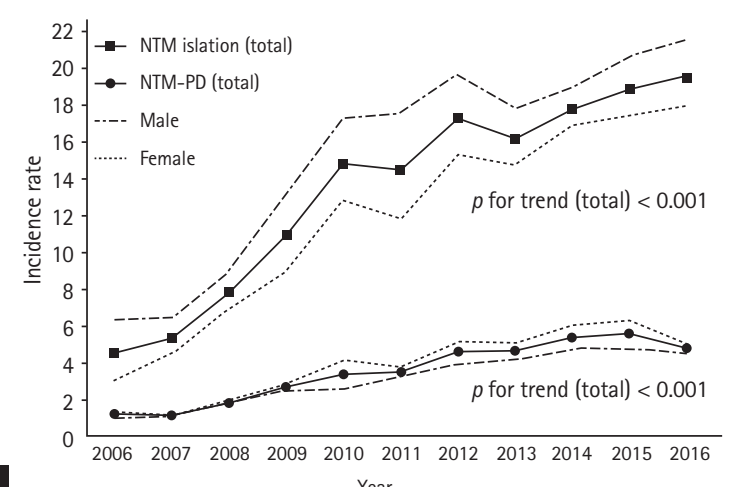

A

Year change in the incidence rate of TB $(88 / 100,000$ in 2006 , and 77/100,000 in 2016) [6,15]. One of the most traditional explanations for this increase is improved detection rates with new laboratory techniques, frequent CT scans during medical check-ups, and increasing awareness of NT'M by the medical staff. However, this detection bias may also be partly due to other reasons. In our institution, the increase in the recovery rate was more prominent for NTM than for M. tuberculosis when we used a combination of liquid and solid media for mycobacterial culture, as has previously been reported [16]. However, when we re-analyzed the mycobacterial recovery rate for the solid culture medium, an increasing trend for NTM isolates and patients with NTM-PD was also evident [7]. Therefore, the increase in the detection of NTM isolates and patients with NTM-PD in this study could not be fully explained by the introduction of the liquid culture

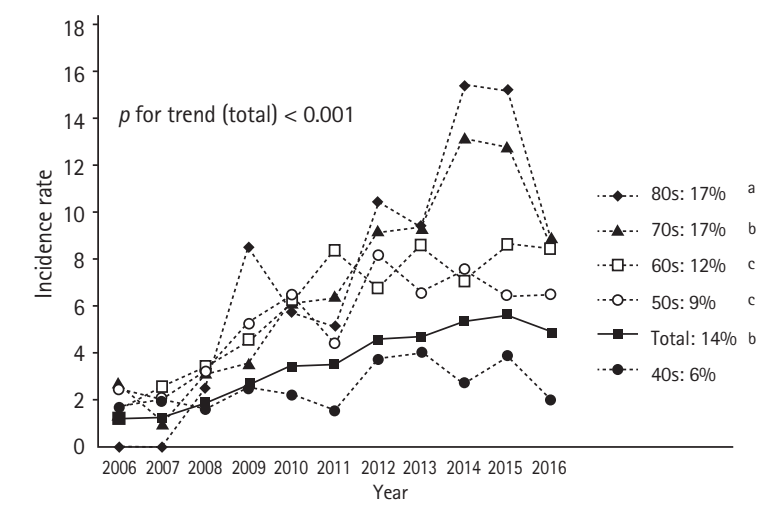

Figure 2. Incidence rate of the isolated nontuberculous mycobacteria (NTM) and NTM pulmonary disease (NTM-PD; per 100,000 person-year). (A) Incidence rate of the patients with isolated NTM and with NTM-PD according to sex. (B) Incidence rate of the patients with NTM-PD according to the age group and its annual increase percent. The $p$ value obtained by Poisson regression analysis: ${ }^{\mathrm{a}} \mathrm{p}<0.05,{ }^{\mathrm{b}} p<0.001$, and ${ }^{\mathrm{c}} p<0.01$.

Table 2. Causative species of NTM-PD according to age and radiologic type

\begin{tabular}{|c|c|c|c|c|c|c|c|}
\hline & $\begin{array}{c}\text { Total } \\
(\mathrm{n}=1,017)\end{array}$ & $\begin{array}{c}\text { Male } \\
(\mathrm{n}=419)\end{array}$ & $\begin{array}{l}\text { Female } \\
(n=598)\end{array}$ & $p$ value & $\begin{array}{l}\text { Fibrocavitary } \\
\qquad(\mathrm{n}=103)\end{array}$ & $\begin{array}{c}\text { Nodular } \\
\text { bronchiectatic } \\
(\mathrm{n}=831)\end{array}$ & $p$ value \\
\hline MAC & $647(63.6)$ & $281(67.1)$ & $366(61.2)$ & 0.065 & $65(63.1)$ & $531(63.9)$ & 0.961 \\
\hline MABC & $110(10.8)$ & $38(9.1)$ & $72(12.0)$ & 0.162 & $10(9.7)$ & $85(10.2)$ & $>0.999$ \\
\hline M. fortuitum & $21(2.1)$ & $12(2.9)$ & $9(1.5)$ & 0.202 & $4(3.9)$ & $15(1.8)$ & 0.148 \\
\hline M. kansasii & $21(2.1)$ & $15(3.6)$ & $6(1.0)$ & 0.009 & $6(5.8)$ & $10(1.2)$ & 0.005 \\
\hline Others & $13(1.3)$ & $11(2.6)$ & $2(0.3)$ & 0.004 & $3(2.9)$ & $9(1.1)$ & 0.137 \\
\hline Multi-species & $205(20.2)$ & $62(14.8)$ & $143(23.9)$ & $<0.001$ & $15(14.6)$ & $181(21.8)$ & 0.117 \\
\hline
\end{tabular}

Values are presented as number (\%).

NTM-PD, nontuberculous mycobacterial pulmonary disease; MAC, Mycobacterium avium complex; MABC, M. abscessus complex. 


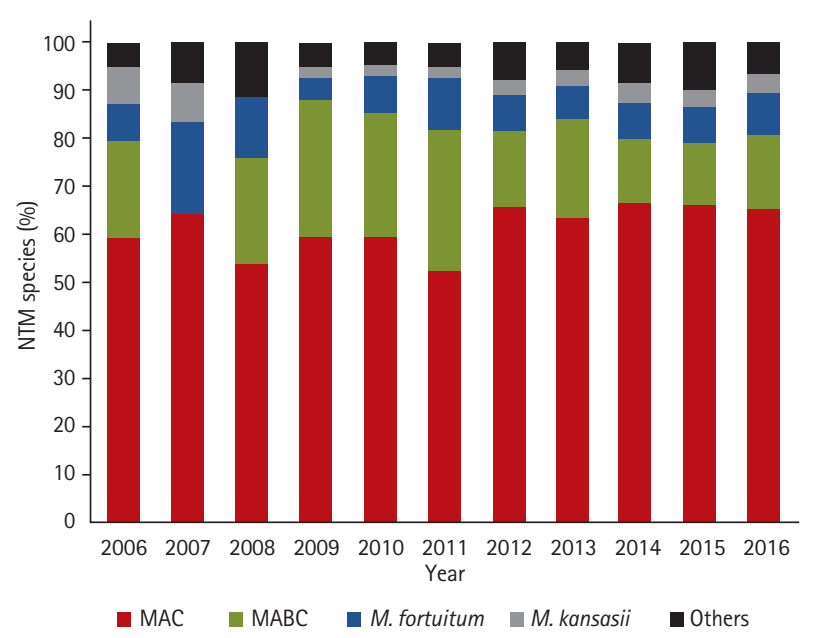

Figure 3. Proportion of the causative nontuberculous mycobacteria (NTM) species in the patients with NTM pulmonary disease (NTM-PD). MAC, Mycobacterium avium complex; MABC, M. abscessus complex.

methods.

We hypothesized that the increase in the incidence rate of NTM-PD was a multifactorial event that includes aging of the general population, an increasing number of patients with comorbidities, and environmental factors. Previous studies have reported that age is related to the increase in the incidence rate of NTM-PD in both young and old populations $[17,18]$. Al-Houqani et al. [19] reported that age is considered to play the major role in the increase of NTM-PD incidence; however, they also stated that age accounted for less than $25 \%$ of the total increase. In the current study, the incidence rate and its increase were more prominent in the old population than in the total population (Fig. 2B). We classified the patients according to age in decades and found an increase in the annual percent change in the incidence rate according to age. Prevots et al. [20] showed similar results; they found a higher increase in the annual prevalence in individuals aged $\geq 60$ years than in those aged $<60$ years. Adjemian et al. [21] also calculated the adjusted odds ratio of the NTM-PD incidence in different age groups compared to that in patients aged $<18$ years, and reported that it is increased according to age: 7.4 (95\% CI, 2.9 to 19.3) in patients aged 18 to 49 years; 31.2 (95\% CI, 12.2 to 79.7 ), patients aged 50 to 64 years; and 106.4 (95\% CI, 42.0 to 270.0), patients aged $\geq 65$ years.

Chronic lung diseases, such as COPD, bronchiectasis, cystic fibrosis, and pneumoconiosis are important pre-

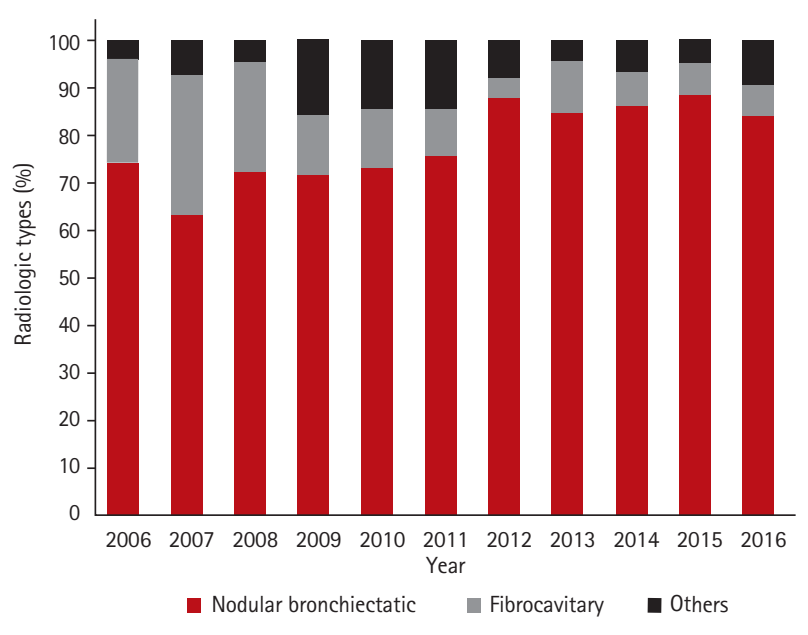

Figure 4. Radiologic types of nontuberculous mycobacterial pulmonary disease.

disposing factors for the development of NTM-PD [4]. The underlying structural lung disease and airway dysfunction may intuitively reduce the clearance of the inhaled environmental microorganisms. The prevalence of bronchiectasis markedly increases with age, and NTM infection easily occurs as a comorbidity with bronchiectasis [22]. In the current study, bronchiectasis (83.9\%) was the most frequently associated chronic lung disease, followed by previous pulmonary TB (48.3\%) and COPD (13.5\%). Furthermore, the proportion of the patients with NB form also increased significantly during the study period (Fig. 4 and Supplementary Fig. 1). It is unclear whether NTM-PD is a cause or the consequence of bronchiectasis, but both notions are supported by accumulated evidence [23]. A history of pulmonary TB and other pulmonary infections can be a risk factor for bronchiectasis and structural lung disease. The increased global burden of COPD and use of corticosteroid inhalation could partly explain the increasing trend of NTM-PD incidence [24,25].

We found an increased number of NTM-PD patients and a stable proportion of patients with MAC-PD, which implies that MAC infection is the main reason for the increasing trends in this study. In South Korea, MAC is the most common organism causing NTM-PD [1]. Ko et al. [12] suggested that the proportion of MAC infection, especially of M. avium, has increased over 15 years in Korea . An American study also revealed an increasing 
trend of MAC infection with relatively stable numbers of all other NTM species over the last 8 years [21].

A few studies have indicated the sex-related differences in the clinical characteristics of NTM-PD. In a French retrospective study, men were younger than women (48.1 years vs. 55.0 years, $p=0.04$ ), and they showed more considerable improvement at 1 year (odds ratio, 2.34; 95\% CI, 1.26 to 8.16) [26]. However, the study included a small number of patients $(n=119)$, and the 1-year mortality rate was higher among men. In a Japanese cross-sectional study that included 11,034 patients with NTM-PD identified from a national database, the incidence rate of NTMPD was higher among women in all age groups except for those aged $\geq 80$ years, which was similar to the results of our study [27]. Holt et al. [28] reported more severe disease in women, evidenced by more cavitation of the lung, lower body mass index, and more extensive treatment history, and a trend towards more marked ventilatory impairment. However, we could not evaluate the severity or treatment history of NTM-PD using our data.

In the current study, women were younger than men, and the peak incidence rate of NTM-PD was in the $50 \mathrm{~s}$ for women, while that in men was in the 7os. Multi-species NTM-PD was more common in women. Comorbidities were also different; bronchiectasis was more common in women, whereas a higher proportion of men had a history of TB and COPD. This indicates that the reason for increasing NTM-PD incidence rate may differ according to the sex. Nevertheless, the data supporting the sex-related difference hypothesis were limited and equivocal; therefore, prospective studies with a larger cohort are needed.

There are a few limitations of this study. First, the study had a retrospective design. We can only assume the correlation between the disease and clinical characteristics. Second, we analyzed the data from a single center. Although a relatively high numbers of samples and patients were included in the investigation, the clinicodemographic characteristics of the patients visiting our hospital might have affected the result. Third, our institution is a referral hospital frequented by patients with comorbidities. Therefore, the data may not represent the general epidemiology in South Korea due to the referral bias. Fourth, environmental factors for each patient were not considered as part of this retrospective study.
In conclusion, the increased incidence rate of NTMPD continued from 2006 to 2016 at a tertiary referral hospital in South Korea, possibly owing to the aging of the general population with comorbidities. Furthermore, there might be some age- and sex-related differences in the clinical characteristics. As the incidence rate of NTM-PD is projected to continue to increase, a prospective evaluation to understand the nature of the disease and the inherited and acquired host factors is warranted.

\section{KEY MESSAGE}

1. The incidence rate of nontuberculous mycobacterial pulmonary disease (NTM-PD) continued to increase from 2006 to 2016 in a tertiary referral hospital in South Korea.

2. The increase in the incidence rate was higher in the elderly population than in the young population.

3. Women were younger than men, and the peak incidence rate of NTM-PD was achieved in the $50 \mathrm{~s}$ and $70 \mathrm{~s}$ in women and men, respectively.

\section{Conflict of interest}

No potential conflict of interest relevant to this article was reported.

\section{REFERENCES}

1. Jeon D. Infection source and epidemiology of nontuberculous mycobacterial lung disease. Tuberc Respir Dis (Seoul) 2019;82:94-101.

2. Griffith DE, Aksamit T, Brown-Elliott BA, et al. An official ATS/IDSA statement: diagnosis, treatment, and prevention of nontuberculous mycobacterial diseases. Am J Respir Crit Care Med 2007;175:367-416.

3. Haworth CS, Banks J, Capstick T, et al. British Thoracic Society Guideline for the management of non-tuberculous mycobacterial pulmonary disease (NTM-PD). BMJ Open Respir Res 2017;4:eooo242.

4. Prevots DR, Marras TK. Epidemiology of human pulmonary infection with nontuberculous mycobacteria: a review. Clin Chest Med 2015;36:13-34. 
5. Hoefsloot W, van Ingen J, Andrejak C, et al. The geographic diversity of nontuberculous mycobacteria isolated from pulmonary samples: an NTM-NET collaborative study. Eur Respir J 2013;42:1604-1613.

6. World Health Organization. Global Tuberculosis Report 2018 (WHO/CDS/TB/2018.20). Geneva (CH): World Health Organization, 2018.

7. Lee SK, Lee EJ, Kim SK, Chang J, Jeong SH, Kang YA. Changing epidemiology of nontuberculous mycobacterial lung disease in South Korea. Scand J Infect Dis 2012;44:733-738.

8. Yoo JW, Jo KW, Kim MN, et al. Increasing trend of isolation of non-tuberculous mycobacteria in a tertiary university hospital in South Korea. Tuberc Respir Dis (Seoul) 2012;72:409-415.

9. Koh WJ, Chang B, Jeong $\mathrm{BH}$, et al. Increasing recovery of nontuberculous mycobacteria from respiratory specimens over a 10-year period in a tertiary referral hospital in South Korea. Tuberc Respir Dis (Seoul) 2013;75:199-204.

10. Ryu YJ, Koh WJ, Daley CL. Diagnosis and treatment of nontuberculous mycobacterial lung disease: clinicians' perspectives. Tuberc Respir Dis (Seoul) 2016;79:74-84.

11. Kim N, Yi J, Chang CL. Recovery rates of non-tuberculous mycobacteria from clinical specimens are increasing in Korean tertiary-care hospitals. J Korean Med Sci 2017;32:1263-1267.

12. Ko RE, Moon SM, Ahn S, et al. Changing epidemiology of nontuberculous mycobacterial lung diseases in a tertiary referral hospital in Korea between 2001 and 2015. J Korean Med Sci 2018;33:e65.

13. Park YS, Lee CH, Lee SM, et al. Rapid increase of non-tuberculous mycobacterial lung diseases at a tertiary referral hospital in South Korea. Int J Tuberc Lung Dis 2010;14:1069-1071.

14. Lee H, Park HJ, Cho SN, Bai GH, Kim SJ. Species identification of mycobacteria by PCR-restriction fragment length polymorphism of the rpoB gene. J Clin Microbiol 2000;38:2966-2971.

15. World Health Organization. Global Tuberculosis Control, 2008 (WHO/HTM/TB/2008.393). Geneva (CH): World Health Organization, 2008.

16. Sorlozano A, Soria I, Roman J, et al. Comparative evaluation of three culture methods for the isolation of mycobacteria from clinical samples. J Microbiol Biotechnol 2009;19:1259-1264.

17. Pierre-Audigier C, Ferroni A, Sermet-Gaudelus I, et al.
Age-related prevalence and distribution of nontuberculous mycobacterial species among patients with cystic fibrosis. J Clin Microbiol 2005;43:3467-3470.

18. Van Ingen J, Hoefsloot W, Dekhuijzen PN, Boeree MJ, van Soolingen D. The changing pattern of clinical Mycobacterium avium isolation in the Netherlands. Int J Tuberc Lung Dis 2010;14:1176-1180.

19. Al-Houqani M, Jamieson F, Mehta M, Chedore P, May K, Marras TK. Aging, COPD, and other risk factors do not explain the increased prevalence of pulmonary Mycobacterium avium complex in Ontario. Chest 2012;141:190-197.

20. Prevots DR, Shaw PA, Strickland D, et al. Nontuberculous mycobacterial lung disease prevalence at four integrated health care delivery systems. Am J Respir Crit Care Med 2010;182:970-976.

21. Adjemian J, Frankland TB, Daida YG, et al. Epidemiology of nontuberculous mycobacterial lung disease and tuberculosis, Hawaii, USA. Emerg Infect Dis 2017;23:439-447.

22. Aksamit TR, O'Donnell AE, Barker A, et al. Adult patients with bronchiectasis: a first look at the US bronchiectasis research registry. Chest 2017;151:982-992.

23. Griffith DE, Aksamit TR. Bronchiectasis and nontuberculous mycobacterial disease. Clin Chest Med 2012;33:283295.

24. World Health Organization. Chronic obstructive pulmonary disease (COPD) [Internet]. Geneva (CH): World Health Organization, c2020 [cited 2020 Jun 3]. Available from: www.who.int/respiratory/copd/burden/en/.

25. Andrejak C, Nielsen R, Thomsen VO, Duhaut P, Sorensen HT, Thomsen RW. Chronic respiratory disease, inhaled corticosteroids and risk of non-tuberculous mycobacteriosis. Thorax 2013;68:256-262.

26. Cadelis G, Ducrot R, Bourdin A, Rastogi N. Predictive factors for a one-year improvement in nontuberculous mycobacterial pulmonary disease: an 11-year retrospective and multicenter study. PLoS Negl Trop Dis 2017;11:e0o05841.

27. Izumi K, Morimoto K, Hasegawa N, et al. Epidemiology of adults and children treated for nontuberculous mycobacterial pulmonary disease in Japan. Ann Am Thorac Soc 2019;16:341-347.

28. Holt MR, Kasperbauer SH, Koelsch TL, Daley CL. Similar characteristics of nontuberculous mycobacterial pulmonary disease in men and women. Eur Respir J 2019;54:1900252. 
KJIM

Supplementary Table 1. Number of patients during the study period

\begin{tabular}{|c|c|c|c|c|c|c|c|}
\hline \multirow{2}{*}{ Year } & \multirow{2}{*}{$\begin{array}{l}\text { Incident cases } \\
\text { of NTM-PD }\end{array}$} & \multicolumn{4}{|c|}{ Pulmonology department } & \multicolumn{2}{|c|}{ Total number } \\
\hline & & Inpatient & Percentage & Outpatient & $\overline{\text { Percentage }}$ & Inpatient & Outpatient \\
\hline 2006 & 25 & 2,166 & 2.81 & 35,612 & 1.85 & 77,091 & $1,928,072$ \\
\hline 2007 & 26 & 2,123 & 2.68 & 36,183 & 1.80 & 79,249 & $2,013,407$ \\
\hline 2008 & 42 & 2,108 & 2.36 & 33,293 & 1.57 & 89,358 & $2,122,755$ \\
\hline 2009 & 66 & 2,169 & 2.32 & 34,036 & 1.51 & 93,295 & $2,255,658$ \\
\hline 2010 & 85 & 1,991 & 2.12 & 33,630 & 1.41 & 93,870 & $2,386,545$ \\
\hline 2011 & 90 & 2,184 & 2.23 & 36,840 & 1.50 & 98,045 & $2,455,259$ \\
\hline 2012 & 120 & 2,071 & 2.05 & 39,030 & 1.55 & 100,995 & $2,523,757$ \\
\hline 2013 & 123 & 2,199 & 2.20 & 40,358 & 1.60 & 99,936 & $2,520,929$ \\
\hline 2014 & 145 & 2,227 & 2.18 & 41,104 & 1.60 & 102,323 & $2,573,775$ \\
\hline 2015 & 155 & 2,617 & 2.42 & 46,699 & 1.76 & 108,340 & $2,650,634$ \\
\hline 2016 & 140 & 2,484 & 2.20 & 50,844 & 1.82 & 112,837 & $2,794,034$ \\
\hline
\end{tabular}

NTM-PD, nontuberculous mycobacterial pulmonary disease. 
Park Y, et al. Rising trend of NTM pulmonary disease

Supplementary Table 2. Age and sex profiles of the incident cases of NTM-PD and incidence rate (per 100,000 person-year) during the study period

\begin{tabular}{|c|c|c|c|c|c|c|c|}
\hline \multirow{3}{*}{$\begin{array}{l}\text { Age group, } \\
\text { yr }\end{array}$} & \multicolumn{4}{|c|}{ Incident cases of NTM-PD } & \multirow{3}{*}{$\begin{array}{l}\text { Rate } \\
\text { ratio }^{\text {a }}\end{array}$} & \multicolumn{2}{|c|}{ Total number } \\
\hline & \multicolumn{2}{|r|}{ Male } & \multicolumn{2}{|r|}{ Female } & & Male & Female \\
\hline & No. (\%) & Incidence rate $(95 \% \mathrm{CI})$ & No. (\%) & Incidence rate (95\% CI) & & Inale & Female \\
\hline $30-39$ & $5(1.2)$ & $0.5(0.2-1.1)$ & $19(3.2)$ & $1.1(0.7-1.8)$ & 2.4 & $1,081,209$ & $1,704,841$ \\
\hline $40-49$ & $13(3.1)$ & $0.9\left(0.5^{-1.6}\right)$ & $74(12.4)$ & $3.5(2.8-4.5)$ & $3 \cdot 9$ & $1,445,775$ & $2,091,878$ \\
\hline $50-59$ & $69(16.5)$ & $3.2(2.5-4.1)$ & $207(34.6)$ & $7.2(6.3-8.3)$ & 2.2 & $2,129,300$ & $2,869,038$ \\
\hline $60-69$ & $143(34.1)$ & $6.1(5.2-7.2)$ & $158(26.4)$ & $6.2(5 \cdot 3-7 \cdot 3)$ & 1.0 & $2,338,416$ & $2,531,907$ \\
\hline $70-79$ & $150(35.8)$ & $9.1(7 \cdot 7-10.7)$ & $108(18.1)$ & $6.8(5.6-8.2)$ & 0.7 & $1,654,679$ & $1,596,645$ \\
\hline$\geq 80$ & $36(8.6)$ & $9.5(6.7-13.2)$ & $28(4.7)$ & $7 \cdot 5(5 \cdot 0-10.9)$ & 0.8 & 380,805 & 375,772 \\
\hline Total & $419(100)$ & $3 \cdot 3(3.0-3.6)$ & $598(100)$ & $4.0(3.7-4.4)$ & 1.2 & $12,798,235$ & $14,829,610$ \\
\hline
\end{tabular}

NTM-PD, nontuberculous mycobacterial pulmonary disease; CI, confidence interval.

${ }^{\mathrm{a}}$ Ratio of female to male incidence rate. 

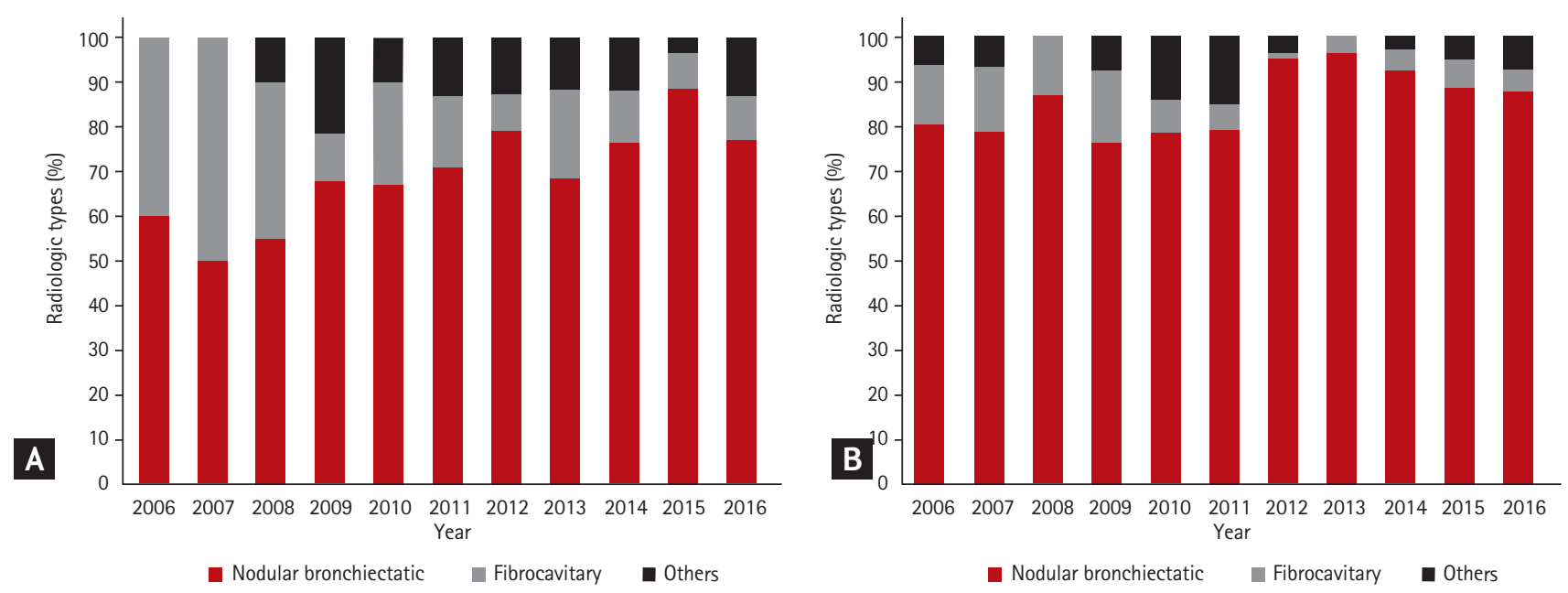

Supplementary Figure 1. Radiologic types of nontuberculous mycobacterial pulmonary disease in (A) male and (B) female patients. 